\title{
MODULI SPACES OF FLAT CONNECTIONS ON 2-MANIFOLDS, COBORDISM, AND WITTEN'S VOLUME FORMULAS
}

\author{
E. MEINRENKEN AND C. WOODWARD
}

\section{INTRODUCTION}

According to Atiyah-Bott $\mathrm{AB}$, $\mathrm{A}$ ] the moduli space of flat connections on a compact oriented 2-manifold with prescribed holonomies around the boundary is a finitedimensional symplectic manifold, possibly singular. A standard approach [W1, W2, प] to computing invariants (symplectic volumes, Riemann-Roch numbers, etc.) of the moduli space is to study the "factorization" of invariants under gluing of 2-manifolds along boundary components. Given such a factorization result, any choice of a "pants decomposition" of the 2-manifold reduces the computation of invariants to the three-holed sphere.

Consider a compact, connected, simple Lie group $G$ with maximal torus $T$, and let $\mathfrak{A} \subset \mathfrak{t}$ be some choice of a fundamental Weyl alcove. We interpret $\mathfrak{A}$ as the set of conjugacy classes in $G$ since for every conjugacy class $\mathcal{C} \subset G$ there is a unique $\mu \in \mathfrak{A}$ with $\exp (\mu) \in \mathcal{C}$. For $\mu_{1}, \mu_{2}, \mu_{3} \in \mathfrak{A}$ let

$$
\mathcal{M}\left(\Sigma_{0}^{3}, \mu_{1}, \mu_{2}, \mu_{3}\right)
$$

denote the moduli space of flat $G$-connections on the three-holed sphere $\Sigma_{0}^{3}$ for which the holonomies around the three boundary components lie in the conjugacy classes labeled by the $\mu_{j}$.

Thanks to the following result of L. Jeffrey [ []], the moduli spaces $\mathcal{M}\left(\Sigma_{0}^{3}, \mu_{1}, \mu_{2}, \mu_{3}\right)$ are well-understood when the holonomies are small. Use the normalized invariant inner product on $\mathfrak{g}$ to identify $\mathfrak{g} \cong \mathfrak{g}^{*}$ and let $\mathcal{O}_{\mu_{j}}$ be the coadjoint orbit through $\mu_{j}$, equipped with the Kostant-Kirillov-Souriau symplectic form, and $\mathcal{O}_{\mu_{j}}^{-}$the same space with the opposite symplectic form.

Theorem 1.1 (Jeffrey). For $\mu_{1}, \mu_{2}, \mu_{3} \in \mathfrak{A}$ sufficiently small, there is a symplectomorphism

$$
\mathcal{M}\left(\Sigma_{0}^{3}, \mu_{1}, \mu_{2}, \mu_{3}\right) \cong \mathcal{O}_{\mu_{1}}^{-} \times \mathcal{O}_{\mu_{2}}^{-} \times \mathcal{O}_{\mu_{3}}^{-} / / G
$$

of the moduli space with a symplectic reduction of a triple product of coadjoint orbits.

In MW2 we prove the following result for more general holonomies:

Date: May 20, 1997.

Supported by a Feodor Lynen fellowship from the Humboldt foundation.

Supported by an NSF Postdoctoral Fellowship. 
Theorem 1.2. For $\mu_{1}, \mu_{2}, \mu_{3} \in \operatorname{int}(\mathfrak{A})$ generic there is an oriented orbifold cobordism

$$
\mathcal{M}\left(\Sigma_{0}^{3}, \mu_{1}, \mu_{2}, \mu_{3}\right) \sim \coprod_{w \in W_{a f f}^{+}}(-1)^{\operatorname{length}(w)}\left(\mathcal{O}_{\mu_{1}}^{-} \times \mathcal{O}_{\mu_{2}}^{-} \times \mathcal{O}_{w \mu_{3}}^{-}\right) / / G .
$$

Here the signs indicate a change in orientation relative to the symplectic orientation, and $W_{\text {aff }}^{+}$is the set of all $w$ in the affine Weyl group $W_{\text {aff }}$ such that $w \mathfrak{A} \subset \mathfrak{t}_{+}$. The symplectic forms extend to a closed 2-form over the cobordism. For $G=\mathrm{SU}(n)$ both sides are smooth manifolds and the cobordism is a manifold cobordism.

The genericity assumption guarantees that both sides have at worst orbifold singularities. Cobordisms of manifolds together with closed 2-forms were introduced and studied by Ginzburg-Guillemin-Karshon [GGK.

The main point of this article is not to give a complete proof of the theorem (which will appear in [MW2]) but rather to explain the main ideas and to present as an application an elementary proof of Witten's formulas for symplectic volumes. These formulas were proved in Witten's original paper [W1]; alternative proofs appear for $\mathrm{SU}(2)$ in [D1, T, JW1, JW2 and for higher rank in [L1, L2, JK]. In a forthcoming paper, we use similar cobordism techniques to compute the Verlinde numbers.

This article is organized as follows. In the following section we introduce the GuilleminGinzburg-Karshon notion of cobordism of Hamiltonian spaces and give some examples. In section 3 we recall the construction of the symplectic form on moduli spaces for 2manifolds with boundary. In section 4 we explain our construction of the cobordism (1), and in section 5 we use (1) to calculate volumes of moduli spaces. In the appendix we collect some background material on Hamiltonian group actions and symplectic reduction.

\section{HAMiltonian COBORDism}

Let $K$ be a compact Lie group. By a Hamiltonian $K$-manifold, we mean a triple $(M, \omega, \Phi)$ consisting of a manifold $M$ with an action of $K$, an invariant closed 2-form $\omega \in \Omega^{2}(M)^{K}$, and an equivariant moment map $\Phi \in C^{\infty}\left(M, \mathfrak{k}^{*}\right)^{K}$ satisfying

$$
\mathrm{d}\langle\Phi, \xi\rangle=\omega\left(\xi_{M}, \cdot\right)
$$

for all $\xi \in \mathfrak{k}$, where $\xi_{M}$ denotes the fundamental vector field.

Introducing the complex $\Omega_{K}(M)=\left(\Omega(M) \otimes S\left(\mathfrak{k}^{*}\right)\right)^{K}$ of equivariant differential forms, with equivariant differential $\tilde{d} \alpha(\xi)=\mathrm{d} \alpha(\xi)-2 \pi i \iota\left(\xi_{M}\right) \alpha(\xi)$, Equation (2) may be rephrased as the cocycle condition for the equivariant 2 -form

$$
\tilde{\omega}=\omega+2 \pi i \Phi .
$$

We denote its equivariant cohomology class by $[\tilde{\omega}] \in H_{K}^{*}(M)$. If $\omega$ is non-degenerate it is called symplectic. In the symplectic case, the Liouville volume is defined as $\operatorname{Vol}(M)=$ $\int_{M} \exp (\omega)$. 
For any $\mu \in \mathfrak{k}^{*}$, the reduced space $M_{\mu}$ is defined as the quotient

$$
M_{\mu}:=\Phi^{-1}(\mu) / K_{\mu} .
$$

For $\mu=0$ we will also use the notation $M_{0}=M / / K$. If $\mu$ is a regular value of $\Phi$, the action of the stabilizer group $K_{\mu}$ on $\Phi^{-1}(\mu)$ is locally free and the reduced space $M_{\mu}$ is an orbifold, with a naturally induced closed 2-form $\omega_{\mu}$. Given a second Hamiltonian action of a Lie group $H$ on $M$ such that the actions of $H$ and $K$ commute, the $K$-reduced space $M_{\mu}$ becomes a Hamiltonian $H$-orbifold. If $\omega$ is symplectic then $\omega_{\mu}$ is symplectic.

The notion of cobordism of Hamiltonian spaces was recently introduced by GuilleminGinzburg-Karshon GGK.

Definition 2.1. GGK Let $\left(M_{1}, \omega_{1}, \Phi_{1}\right)$ and $\left(M_{2}, \omega_{2}, \Phi_{2}\right)$ be two oriented Hamiltonian $K$-manifolds with proper moment maps. We call $M_{1}$ and $M_{2}$ cobordant and write $M_{1} \sim M_{2}$ if there exists an oriented Hamiltonian $K$-manifold with boundary $(N, \omega, \Phi)$ with proper moment map such that $\partial N=M_{1} \cup\left(-M_{2}\right)$ and such that $\omega$ resp. $\Phi$ pull back to $\omega_{i}$ resp. $\Phi_{i}$.

(The properness assumption on the moment map is crucial since otherwise this notion of cobordism would be more or less void.) There is an analogous definition of cobordisms of Hamiltonian orbifolds.

One is mainly interested in the case that the "ends" of the cobordism are symplectic. Their orientation, however, need not agree with the symplectic orientation. Letting $M_{j, r}$ denote the connected components of $M_{j}$, we have as an obvious cobordism invariant the signed symplectic volume:

$$
\sum_{r} \pm \operatorname{Vol}\left(M_{1, r}\right)=\sum_{r} \pm \operatorname{Vol}\left(M_{2, r}\right)
$$

where the signs are +1 or -1 according whether or not the orientation agrees with the symplectic orientation.

The simplest example of a Hamiltonian cobordism is provided by two cohomologous equivariant 2 -forms $\tilde{\omega}_{j}=\omega_{j}+2 \pi i \Phi_{j}$ on a compact oriented $G$-manifold $M$. Thus suppose $M_{1}=M_{2}=M$ and that there exists a 1 -form $\beta \in \Omega^{1}(M)^{K}$ such that

$$
\omega_{2}-\omega_{1}=\mathrm{d} \beta, \Phi_{2}-\Phi_{1}=-\beta^{\sharp}
$$

where $\beta^{\sharp}: M \rightarrow \mathfrak{g}^{*}$ is defined by $\left\langle\beta^{\sharp}, \xi\right\rangle=\iota\left(\xi_{M}\right) \beta$. Let $N=M \times[0,1]$ with points denoted $(m, t)$, and let

$$
\omega=\omega_{1}+\mathrm{d}(t \beta), \Phi(m, t)=(1-t) \Phi_{1}(m)+t \Phi_{2}(m) .
$$

Then $(N, \omega, \Phi)$ provides a cobordism of Hamiltonian $K$-manifolds, $M_{1} \sim M_{2}$.

One of the main results in GGK is that any compact symplectic Hamiltonian $T$ manifold with $(M, \omega, \Phi)$ with isolated fixed points is cobordant in the above sense to its "polarized" linearization around the fixed points. More precisely, let $a_{j}(p) \in \mathfrak{t}^{*}$, 
$j=1, \ldots, n=\frac{1}{2} \operatorname{dim} M$ be the weights for the $T$-action at the fixed points. Thus $T_{p} M \cong \mathbb{C}^{n}$, and the induced $T$-action has moment map

$$
\Psi_{p}: T_{p} M \rightarrow \mathfrak{t}^{*}, z \mapsto \Phi(p)-\frac{1}{2} \sum_{j}\left|z_{j}\right|^{2} a_{j}(p) .
$$

Choose a vector $\xi \in \mathfrak{t}$ that is not perpendicular to any of the weights $a_{j}(p)$. Let

$$
a_{j}^{\sharp}(p)=\operatorname{sign}\left\langle a_{j}(p), \xi\right\rangle a_{j}(p)
$$

denote the polarized weights and $\sigma_{j}(p)=\#\left\{j \mid\left\langle a_{j}(p), \xi\right\rangle<0\right\}$ the number of sign changes. Let $T_{p} M^{\sharp}$ denote the tangent space, with the modified $T$-action with moment map

$$
\Psi_{p}^{\sharp}: z \mapsto \Phi(p)-\frac{1}{2} \sum_{j}\left|z_{j}\right|^{2} a_{j}^{\sharp}(p) .
$$

Theorem 2.2. GGK For any compact symplectic Hamiltonian T-manifold $M$ with isolated fixed points, there is a cobordism of Hamiltonian T-manifolds

$$
M \sim \coprod_{p \in M^{T}}(-1)^{\sigma(p)} T_{p} M^{\sharp} .
$$

If $M_{1} \sim M_{2}$ is a cobordism of Hamiltonian $K$-manifolds, and $\nu \in \mathfrak{k}^{*}$ a regular value of the moment map for the cobordism, reduction gives rise to a cobordism $\left(M_{1}\right)_{\nu} \sim\left(M_{2}\right)_{\nu}$. Using a simple perturbation argument, GGK show that if $K_{\nu}$ is a maximal torus it is in fact sufficient to assume that $\nu$ is a regular value for the moment maps $\Phi_{1}, \Phi_{2}$.

For example, in Theorem 2.2 we have a cobordism

$$
M_{\nu} \sim \coprod_{p \in M^{T}}(-1)^{\sigma(p)}\left(T_{p} M^{\sharp}\right)_{\nu}
$$

As an immediate consequence one obtains the formula of Guillemin-Lerman-Sternberg GLS

$$
\operatorname{Vol}\left(M_{\nu}\right)=\sum(-1)^{\sigma(p)} \frac{\# \Gamma_{p}}{\operatorname{Vol}(T)} \kappa_{p}(\Phi(p)-\nu),
$$

where $\kappa_{p}$ is the push-forward of the characteristic measure on the positive orthant $\mathbb{R}_{+}^{n}$ under the map

$$
\mathbb{R}^{n} \rightarrow \mathfrak{t}^{*}, x \mapsto \sum_{j=1}^{n} x_{j} a_{j}^{\sharp}(p),
$$

$\Gamma_{p}$ the generic stabilizer for the action on $T_{p} M^{\sharp}$, and $\operatorname{Vol}(T)$ the volume with respect to an inner product on $\mathfrak{t}$ which we also use to identify measures and functions.

Example 2.3. Let $T$ be the maximal torus of $K$ and $\mathfrak{t}_{+}^{*} \subset \mathfrak{t}^{*} \subset \mathfrak{g}^{*}$ some choice of a positive Weyl chamber. Let $\mu \in \operatorname{int}\left(\mathfrak{t}_{+}^{*}\right)$ and $\mathcal{O}_{\mu}=K \cdot \mu \cong K / T$ the coadjoint orbit through $\mu$, equipped with the KKS symplectic form $\sigma_{\mu} \in \Omega^{2}\left(\mathcal{O}_{\mu}\right)$. The moment map for the $K$-action on $\mathcal{O}_{\mu}$ is the embedding $\iota_{\mu}: \mathcal{O}_{\mu} \hookrightarrow \mathfrak{k}^{*}$. Let $\mathcal{O}_{\mu}^{(T)}$ denote the orbit 
considered as a $T$-manifold, with moment map $\operatorname{pr}_{t^{*}} \circ \iota_{\mu}$. The $T$-fixed points are just the Weyl conjugates $w \cdot \mu$, and taking the polarizing vector $\xi \operatorname{in} \operatorname{int}\left(\mathfrak{t}_{+}\right)$the polarized weights are the positive roots. Thus

$$
\operatorname{Vol}\left(\mathcal{O}_{\mu}^{(T)}\right)_{\nu}=\frac{\# Z(G)}{\operatorname{Vol}(T)} \sum_{w \in W}(-1)^{\operatorname{length}(w)} \kappa(w \mu-\nu)
$$

where $\kappa$ is the measure determined by the positive roots. This formula is the classical analogue of the Kostant multiplicity formula.

Example 2.4. Suppose $(M, \omega, \Phi)$ is a compact Hamiltonian $K$-manifold, and $\mu \in \mathfrak{t}_{+}^{*}$. Let $X_{1}=\mathcal{O}_{\mu} \times M$ be the product, with diagonal $K$-action and $\omega_{X_{1}}=\sigma_{\mu}+\omega_{M}$. Let $X_{2}$ be the associated bundle

$$
X_{2}=K \times_{T} M \text {. }
$$

There exists a unique closed 2-form $\omega_{X_{2}}$ on $X_{2}$ for which the $K$-action is Hamiltonian, with moment map $\Phi_{X_{2}}$, and such that the pull-back of $\omega_{X_{2}}$ resp. $\Phi_{X_{2}}$ to $M$ is given by $\omega$ and $\mathrm{pr}_{\mathfrak{t}^{*}} \circ \Phi+\mu$. There is a $K$-equivariant diffeomorphism

$$
\phi: X_{2}=K \times_{T} M \rightarrow X_{1}=\mathcal{O}_{\mu} \times M,[k, m] \mapsto(k \cdot \mu, k \cdot m) .
$$

We leave it as an exercise to the reader to verify that the pull-back $\phi^{*} \tilde{\omega}_{X_{1}}$ of the equivariant symplectic form on $X_{1}$ is equivariantly cohomologous to the equivariant form $\tilde{\omega}_{X_{2}}$. It follows that for any regular value $\tau \in \operatorname{int}\left(\mathfrak{t}_{+}^{*}\right)$ of the moment maps of both $X_{1}$ and $X_{2}$, there is an oriented orbifold cobordism of reduced spaces $\left(\mathcal{O}_{\mu} \times M\right)_{\tau} \sim\left(K \times_{T} M\right)_{\tau}$. The reduction on the right hand side is easily determined: Since $\Phi_{X_{2}}([k, m])=k \cdot\left(\mu+\operatorname{pr}_{t^{*}} \circ \Phi\right)$, the $\tau$-level set condition is $\operatorname{pr}_{\mathfrak{t}^{*}} \circ \Phi=k^{-1} \tau-\mu$. Since this is contained in $\mathfrak{t}^{*}$ only if $k^{-1}$ represents an element $w$ of the Weyl group, it follows that the reduction is a disjoint union of $T$-reductions of $M$ at values $w \tau-\mu$. Denoting by $M^{(T)}$ the space $M$ considered as a Hamiltonian $T$-manifold, we find a cobordism

$$
\left(\mathcal{O}_{\mu} \times M\right)_{\tau} \sim \coprod_{w \in W}(-1)^{\text {length }(w)} M_{w \tau-\mu}^{(T)}
$$

The resulting formula

$$
\operatorname{Vol}\left(\mathcal{O}_{\mu} \times M\right)_{\tau}=\sum_{w \in W}(-1)^{\operatorname{length}(w)} \operatorname{Vol}\left(M_{w \tau-\mu}^{(T)}\right)
$$

may be considered the classical analogue to the Frobenius reciprocity formula. Specializing to the case where $M=\mathcal{O}_{\nu}$ is a coadjoint orbit $\left(\nu \in \operatorname{int}\left(\mathfrak{t}_{+}^{*}\right)\right)$ and combining with the previous example we find a cobordism

$$
\left(\mathcal{O}_{\mu} \times \mathcal{O}_{\nu}\right)_{\tau} \sim \coprod_{w_{1} \in W} \sum_{w_{2} \in W}(-1)^{\text {length }\left(w_{1} w_{2}\right)}(\mathfrak{k} / \mathfrak{t})_{w_{1} \mu+w_{2} \nu-\tau}
$$


where $\mathfrak{k} / \mathfrak{t}$ has symplectic structure given by any $T$-invariant Hermitian metric."] As a consequence we have for symplectic volumes the formula

$$
\operatorname{Vol}\left(\mathcal{O}_{\mu} \times \mathcal{O}_{\nu}\right)_{\tau}=\frac{\# Z(G)}{\operatorname{Vol}(T)} \sum_{w_{1} \in W} \sum_{w_{2} \in W}(-1)^{\operatorname{length}\left(w_{1} w_{2}\right)} \kappa\left(w_{1} \mu+w_{2} \nu-\tau\right)
$$

which is the classical analogue of the Steinberg formula. This was derived in GuilleminPrato [GP] by a different method.

\section{Moduli Spaces of Flat Connections}

In this section we recall the construction of moduli spaces of flat connections over 2-manifolds. Let $G$ be a simple, compact, connected Lie group and $T \subset G$ its maximal torus. The integral lattice $\{\xi \in \mathfrak{t} \mid \exp (\xi)=1\}$ will be denoted by $\Lambda$. We let the weight lattice $\Lambda^{*} \subset \mathfrak{t}^{*}$ be its dual and let $\mathfrak{R}^{+} \subset \Lambda^{*}$ be a system of positive roots of $G$. I Denote by $\mathfrak{t}_{+} \subset \mathfrak{t}$ the positive Weyl chamber determined by $\mathfrak{R}_{+}$and by

$$
\mathfrak{A}=\left\{\xi \in \mathfrak{t}_{+} \mid\left\langle\alpha_{\max }, \xi\right\rangle \leq 1\right\}
$$

the closed fundamental alcove, where $\alpha_{\max } \in \mathfrak{R}^{+}$is the highest root. We identify $\mathfrak{A}$ with the set $\operatorname{Conj}(G)$ of conjugacy classes of $G$ via

$$
\mathfrak{A} \cong \mathfrak{t} / W_{\text {aff }} \cong T / W \cong G / \operatorname{Ad}(G)=\operatorname{Conj}(G),
$$

where $W$ is the Weyl group and $W_{\text {aff }}=\Lambda \rtimes W$ the affine Weyl group.

Consider a compact, connected, oriented 2-manifold $\Sigma=\Sigma_{h}^{b}$ of genus $h$ with $b$ boundary components $B_{1}, \ldots, B_{b}$. Let $\mathcal{A}(\Sigma) \cong \Omega^{1}(\Sigma, \mathfrak{g})$ denote the space of connections on the trivial bundle $\Sigma \times G$. We take these connections to be of a fixed Sobolev class $r>\frac{1}{2}$ and consider $\mathcal{A}(\Sigma)$ as a Banach manifold. The gauge group $\mathcal{G}(\Sigma)=\operatorname{Map}(\Sigma, G)$ consisting of maps of Sobolev class $r+1$ is a Banach Lie group, which acts on $\mathcal{A}(\Sigma)$ by $g \cdot A=\operatorname{Ad}_{g}(A)-\mathrm{d} g g^{-1}$. For any boundary $B_{j}$ and any connection $A \in \mathcal{A}(\Sigma)$, the holonomy $\operatorname{Hol}_{B_{j}}(A)$ around $B_{j}$ is well-defined up to conjugacy; here we take the orientation of the boundary $\partial \Sigma$ to be opposite to the orientation induced from $\Sigma$. Given conjugacy classes $\mathcal{C}_{1}, \ldots, \mathcal{C}_{b} \subset G$ labeled by $\mu_{1}, \ldots, \mu_{b} \in \mathfrak{A}$, let

$$
\mathcal{M}\left(\Sigma, \mu_{1}, \ldots, \mu_{b}\right):=\left\{A \in \mathcal{A}(\Sigma) \mid \operatorname{curv}(A)=0, \operatorname{Hol}_{B_{j}}(A) \in \mathcal{C}_{j}\right\} / \mathcal{G}(\Sigma)
$$

be the moduli space of flat connections with holonomies in $\mathcal{C}_{j}$. (This space is independent of the choice of Sobolev class $r$.) There is a canonical isomorphism between this space

\footnotetext{
${ }^{1}$ Any two such structures are equivariantly symplectomorphic.

${ }^{2}$ Note that we work with real weights as in BrD. Our main motivation for this convention is that the "pre-quantizable" coadjoint orbits $G \cdot \mu,\left(\mu \in \mathfrak{t}^{*} \subset \mathfrak{g}^{*}\right)$ are precisely those through real weight lattice points. By contrast Witten W1, W2 works with infinitesimal roots and weights, these differ by a factor $2 \pi i$ and give rise to different powers of $2 \pi$ in some of his formulas.
} 
and the representation variety

$$
\left\{(a, c) \in G^{2 h} \times \mathcal{C}_{1} \times \ldots \times \mathcal{C}_{b} \mid \prod_{i=1}^{h}\left[a_{2 i-1}, a_{2 i}\right]=\prod_{j=1}^{b} c_{j}\right\} / G
$$

given by describing each gauge equivalence class in terms of its parallel transport. This description shows that $\mathcal{M}\left(\Sigma, \mu_{1}, \ldots, \mu_{b}\right)$ is a compact, stratified space (usually singular). It was one of the basic observations in Atiyah-Bott [AB] that the spaces $\mathcal{M}\left(\Sigma, \mu_{1}, \ldots, \mu_{b}\right)$ carry natural symplectic structures, and that (16) can in fact be viewed as a symplectic quotient. Indeed, $\mathcal{A}(\Sigma)$ carries a natural symplectic form

$$
\omega_{A}\left(a_{1}, a_{2}\right)=\int_{\Sigma} a_{1} \wedge a_{2} \quad\left(a_{i} \in T_{A} \mathcal{A}(\Sigma) \cong \Omega^{1}(\Sigma, \mathfrak{g})\right),
$$

using the normalized invariant inner product on $\mathfrak{g}$ for which $\alpha_{\max }$ has length $\sqrt{2}$. 3 The $\mathcal{G}(\Sigma)$-action preserves the symplectic form and is in fact Hamiltonian, with moment map given by

$$
\Psi(A)=(\operatorname{curv}(A), A \mid \partial \Sigma) \in \Omega^{2}(\Sigma, \mathfrak{g}) \oplus \Omega^{1}(\partial \Sigma, \mathfrak{g}),
$$

that is

$$
\langle\Psi(A), \xi\rangle=\int_{\Sigma} \operatorname{curv}(A) \cdot \xi+\int_{\partial \Sigma} \iota^{*}(A \cdot \xi)
$$

for $\xi \in \Omega^{0}(\Sigma, \mathfrak{g})=\operatorname{Lie}(\mathcal{G}(\Sigma))$. This exhibits $\mathcal{M}\left(\Sigma, \mu_{1}, \ldots, \mu_{b}\right)$ as a symplectic reduction since the pull-backs $A \mid B_{j}$ are determined up to gauge equivalence by the conjugacy class of their holonomies $\mathrm{Hol}_{B_{j}}(A)$.

In the presence of at least one boundary component, it is convenient to perform the above reduction in stages. Let $\mathcal{G}(\partial \Sigma)=\operatorname{Map}(\partial \Sigma, G)$ (maps of Sobolev class $r+\frac{1}{2}$ ) be the gauge transformations of the boundary. Since $G$ is simply connected, the restriction map $\mathcal{G}(\Sigma) \rightarrow \mathcal{G}(\partial \Sigma)$ is surjective. Its kernel $\mathcal{G}_{\partial}(\Sigma)$ consists of gauge transformations that are the identity on the boundary. The moment map for the action of $\mathcal{G}_{\partial}(\Sigma)$ on $\mathcal{A}(\Sigma)$ is just the curvature $A \rightarrow \operatorname{curv}(A)$. Let $\mathcal{M}(\Sigma)$ be the symplectic quotient

$$
\mathcal{M}(\Sigma)=\mathcal{A}(\Sigma) / / \mathcal{G}_{\partial}(\Sigma)=\{A \in \mathcal{A}(\Sigma) \mid \operatorname{curv}(A)=0\} / \mathcal{G}_{\partial}(\Sigma) .
$$

Donaldson proved in $[\mathrm{D} 1]$ that if the boundary of $\Sigma$ is non-empty $(b \geq 1)$, the space $\mathcal{M}(\Sigma)$ is a smooth (!) symplectic Banach manifold. The residual action of $\mathcal{G}(\partial \Sigma)=\mathcal{G}(\Sigma) / \mathcal{G}_{\partial}(\Sigma)$ is Hamiltonian, with moment map given by $[A] \rightarrow \iota_{\partial \Sigma}^{*} A \in \Omega^{1}(\partial \Sigma, \mathfrak{g})$.

Choose orientation preserving parametrizations $B_{j} \cong S^{1}$ of the boundary components, thereby identifying the gauge group $\mathcal{G}\left(B_{j}\right)$ with the loop group $L G=\operatorname{Map}\left(S^{1}, G\right)$ and $\Omega^{1}\left(B_{j}, \mathfrak{g}\right)$ with $L \mathfrak{g}^{*}:=\Omega^{1}\left(S^{1}, \mathfrak{g}\right)$. Thus $\mathcal{M}(\Sigma)$ is an example of a Hamiltonian $L G^{b}-$ manifold with proper moment map $\Phi: \mathcal{M}(\Sigma) \rightarrow\left(L \mathfrak{g}^{*}\right)^{b}$. It admits a description in

\footnotetext{
${ }^{3}$ This inner product is related to the Killing form by $\langle\xi, \eta\rangle=-\left(8 \pi^{2} n_{G}\right)^{-1} \operatorname{tr}\left(\operatorname{ad}_{\xi} \operatorname{ad}_{\eta}\right)$, where $n_{G}$ is the dual Coxeter number of $G$.
} 
terms of holonomies, just as the spaces $\mathcal{M}\left(\Sigma, \mu_{1}, \ldots, \mu_{b}\right)$ :

$$
\mathcal{M}(\Sigma)=\left\{(a, c, \zeta) \in G^{2 g} \times G^{b-1} \times\left(L \mathfrak{g}^{*}\right)^{b} \mid \prod_{i=1}^{2 g}\left[a_{2 i-1}, a_{2 i}\right]=\prod_{i=1}^{b} \operatorname{Ad}_{c_{i}} \operatorname{Hol}\left(\zeta_{i}\right)\right\}
$$

where $c_{1}=1$. In this description the action of $g=\left(g_{1}, \ldots, g_{b}\right) \in L G^{b}$ is given by

$$
g \cdot a_{i}=\operatorname{Ad}_{g_{1}(0)} a_{i}, \quad g \cdot c_{j}=g_{1}(0) c_{j} g_{j}(0)^{-1}, \quad g \cdot \zeta_{j}=\operatorname{Ad}_{g_{j}} \cdot \mu_{j}-\mathrm{d} g_{j} g_{j}{ }^{-1}
$$

and the moment map is the projection to the $\left(L \mathfrak{g}^{*}\right)^{b}$-factor.

Note that the moment map is equivariant with respect to the affine action of $L G$ on $L \mathfrak{g}^{*}$ which corresponds to interpreting $L \mathfrak{g}^{*}$ as connections on $S^{1}$ and $L G$ as the gauge group.t The basic examples are as follows:

Example 3.1. a. The moduli space $\mathcal{M}\left(\Sigma_{0}^{1}\right)$ of the 1-holed sphere is the based loop group $\Omega G=L G / G$. The moment map sends $h \in \Omega G$ to $h \cdot 0=-h^{-1} \mathrm{~d} h \in L \mathfrak{g}^{*}$.

b. The moduli space $\mathcal{M}\left(\Sigma_{0}^{2}\right)$ of the 2 -holed sphere is $L G \times L \mathfrak{g}^{*}$. The moment map is $(h, \zeta) \rightarrow(h \cdot \zeta,-\zeta)$.

The Hamiltonian loop group spaces $\mathcal{M}(\Sigma)$ have been studied from various points of view by Donaldson [D1], Segal [S], S. Chang [Q] and others. They satisfy the following factorization property, which we learned from S. Martin [M]:

Theorem 3.2. Let $\Sigma$ be obtained from a possibly disconnected 2-manifold $\hat{\Sigma}$ by gluing two boundary components $B_{ \pm} \subset \partial \hat{\Sigma}$. Let $L G$ act on $\mathcal{M}(\hat{\Sigma})$ by the diagonal action corresponding to these two boundary circles; here we reverse the orientation on $B_{-}$so that the $B_{-}$-moment map is $[\hat{A}] \rightarrow-\iota_{B_{-}}^{*} \hat{A}$. The moduli space $\mathcal{M}(\Sigma)$ is given by symplectic reduction

$$
\mathcal{M}(\Sigma)=\mathcal{M}(\hat{\Sigma}) / / L G .
$$

This follows formally because the zero level condition is that the restrictions $\hat{A} \mid B_{ \pm}$ match up. A more detailed proof is given in MW1.

We view $\mathfrak{g}$ as a subset of $L \mathfrak{g}^{*}$ by identifying $\mu \in \mathfrak{g}$ with the connection $\mu \frac{d \phi}{2 \pi} \in \Omega^{1}\left(S^{1}, \mathfrak{g}\right)$, so that the diagram

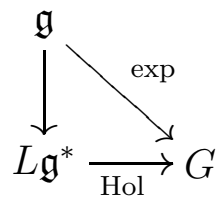

commutes. The moduli spaces $\mathcal{M}\left(\Sigma_{h}^{b}, \mu_{1}, \ldots, \mu_{b}\right)$ with fixed holonomies are obtained from the Hamiltonian $L G^{b}$-manifold $\mathcal{M}\left(\Sigma_{h}^{b}\right)$ as symplectic reductions: Given $\mu_{j} \in \mathfrak{A} \subset$ $\mathfrak{t} \subset \mathfrak{g} \subset L \mathfrak{g}^{*}$ we have

$$
\mathcal{M}\left(\Sigma_{h}^{b}, \mu_{1}, \ldots, \mu_{b}\right)=\mathcal{M}\left(\Sigma_{h}^{b}\right)_{\mu_{1}, \ldots, \mu_{b}}=\Phi^{-1}\left(\mu_{1}, \ldots, \mu_{b}\right) /(L G)_{\mu_{1}} \times \ldots \times(L G)_{\mu_{b}} .
$$

\footnotetext{
${ }^{4}$ Passing to the central extension $\widehat{L G}$ makes this into a linear action, where $L \mathfrak{g}^{*}$ is identified with the affine subspace $L \mathfrak{g}^{*} \times\{1\} \subset \widehat{L \mathfrak{g}}^{*}$. Thus $\mathcal{M}(\Sigma)$ can also be viewed as a Hamiltonian $\widehat{L G}^{b}$-manifold where the central circle(s) acts with constant moment map 1.
} 
To achieve a better understanding of these spaces we need to recall a few elementary facts about the affine coadjoint action of $L G$ on $L \mathfrak{g}^{*}$. The fact that every connection on $S^{1}$ is determined up to gauge equivalence by the conjugacy class of its holonomy means that

$$
L \mathfrak{g}^{*} / L G=G / \operatorname{Ad}(G)=\mathfrak{A} .
$$

That is, every coadjoint $L G$-orbit passes through exactly one point of the fundamental alcove $\mathfrak{A}$. For any $\mu \in \mathfrak{A}$, the stabilizer group $(L G)_{\mu}$ is compact, and depends only on the open face $\sigma$ of $\mathfrak{A}$ containing $\sigma$. It is isomorphic (via the map $g \mapsto g(1)$ ) to the centralizer in $G$ of $\exp (\mu)$.

The affine coadjoint $L G$-action admits finite dimensional slices which can be constructed as follows. Let $\mathfrak{A}_{\sigma}$ be the union of all open faces $\tau$ of $\mathfrak{A}$ that contain $\sigma$ in their closure; thus $(L G)_{\nu} \supset(L G)_{\mu}$ for $\nu \in \mathfrak{A}_{\sigma}$. Then $U_{\sigma}:=(L G)_{\mu} \cdot \mathfrak{A}_{\sigma}$ is a slice for the action at $\mu$.

Proposition 3.3. Let $\sigma_{j} \subset \mathfrak{A}$ be open faces containing $\mu_{j}$. The pre-image

$$
\Phi^{-1}\left(U_{\sigma_{1}} \times \ldots \times U_{\sigma_{b}}\right) \subset \mathcal{M}\left(\Sigma_{h}^{b}\right)
$$

is a smooth, finite dimensional symplectic submanifold, and is a Hamiltonian $(L G)_{\mu_{1}} \times$ $\ldots \times(L G)_{\mu_{b}}$-manifold with the restriction of $\Phi$ serving as a moment map. The moduli space (G) is obtained from (G) as a finite dimensional reduction at $\mu_{1}, \ldots, \mu_{b}$.

This follows from the symplectic cross-section theorem for Hamiltonian $L G$-manifolds with proper moment maps. (See e.g. MW1 for a proof.) Note that the restriction of $\Phi$ is only affine-equivariant if at least one of the $\mu_{j}$ is contained in the closed face of $\mathfrak{A}$ opposite to $\{0\}$. Shifting by the vector $\left(\mu_{1}, \ldots, \mu_{b}\right) \in\left(L \mathfrak{g}^{*}\right)^{b}$ makes it into an equivariant moment map. The finite dimensional spaces (9) are generalized versions of the extended moduli spaces introduced by Chang, Huebschmann and Jeffrey.

The fact that the moduli spaces (6) can be obtained as finite-dimensional reductions has a number of implications. First of all, it shows that they are stratified symplectic spaces in the sense of Sjamaar-Lerman [SD (see Appendix A.1), and therefore have well-defined symplectic volumes. We also know that the function

$$
\left(\mu_{1}, \ldots, \mu_{b}\right) \mapsto \operatorname{Vol}\left(\mathcal{M}\left(\Sigma_{h}^{b}, \mu_{1}, \ldots, \mu_{b}\right)\right)
$$

is continuous and piecewise polynomial over $\Phi\left(\mathcal{M}\left(\Sigma_{h}^{b}\right)\right) \cap \operatorname{int}(\mathfrak{A})^{b}$ (see Appendix A.2). Another consequence is that if $\left(\nu_{1}, \ldots, \nu_{b}\right) \in \mathfrak{A}^{b}$ is a regular value and $\left(\mu_{1}, \ldots, \mu_{b}\right) \in \mathfrak{A}^{b}$ sufficiently close to $\left(\nu_{1}, \ldots, \nu_{b}\right)$, there is a symplectic fibration

$$
\mathcal{M}\left(\Sigma_{h}^{b}, \mu_{1}, \ldots, \mu_{b}\right) \rightarrow \mathcal{M}\left(\Sigma_{h}^{b}, \nu_{1}, \ldots, \nu_{b}\right)
$$

with fiber a product of coadjoint orbits,

$$
(L G)_{\nu_{1}} \cdot\left(\mu_{1}-\nu_{1}\right) \times \ldots \times(L G)_{\nu_{b}} \cdot\left(\mu_{b}-\nu_{b}\right) \subset L \mathfrak{g}_{\nu_{1}}^{*} \times \ldots \times L \mathfrak{g}_{\nu_{b}}^{*}
$$

(see Appendix A.3). 


\section{Construction of the Cobordism}

Instead of reducing with respect to all $L G$-factors at once, we can also reduce with respect to only some of the factors. This is important because the action of $(L G)^{b-1} \subset$ $(L G)^{b}$ on $\mathcal{M}\left(\Sigma_{h}^{b}\right)$ is always free, so that the corresponding reduction is always regular. Reduced spaces near a given value of the moment map are fiber bundles over the reduced space at that value. This would be automatic for finite-dimensional Hamiltonian $K$ manifolds (see Appendix A.3) and is shown for Hamiltonian $L G$-manifolds in [MW2].

Let us apply this to the case of the three-holed sphere. We wish to understand the reductions $\mathcal{M}\left(\Sigma_{0}^{3}\right)_{\mu_{1}, \mu_{2}}$, for $\mu_{j}$ small. From the holonomy description (8), we have

$$
\mathcal{M}\left(\Sigma_{0}^{3}\right)_{0,0, \cdot}=\Omega G=L G / G
$$

as a Hamiltonian $L G$-manifold (alternatively this also follows from the "Gluing equals Reduction" principle, Theorem 3.2). The holonomy description tells us furthermore that the zero level set for the $L G^{2} \subset L G^{3}$-action is an associated bundle $L G \times_{G}(G \times G)$.

Since the coadjoint orbit $(L G)_{0} \cdot \mu$ through a small value $\mu \in \mathfrak{A}$ is just the usual coadjoint orbit $\mathcal{O}_{\mu}$ for $G$, we obtain the following description of the nearby reductions $\mathcal{M}\left(\Sigma_{0}^{3}\right)_{\mu_{1}, \mu_{2}, .}$. Let $L G_{0}=G$ act on the product of coadjoint $G$-orbits $\mathcal{O}_{\mu_{1}}^{-} \times \mathcal{O}_{\mu_{2}}^{-}$by the diagonal action, with moment map

$$
\mathcal{O}_{\mu_{1}}^{-} \times \mathcal{O}_{\mu_{2}}^{-} \ni\left(\alpha_{1}, \alpha_{2}\right) \mapsto-\left(\alpha_{1}+\alpha_{2}\right) \in \mathfrak{g}^{*}
$$

The principal bundle $G \rightarrow L G \rightarrow \Omega G$ has a canonical $L G$-invariant connection coming from the splitting of Lie algebras $L \mathfrak{g}=\mathfrak{g} \oplus \Omega \mathfrak{g}$. The minimal coupling form on the associated bundle $L G \times{ }_{G}\left(\mathcal{O}_{\mu_{1}}^{-} \times \mathcal{O}_{\mu_{2}}^{-}\right)$defined by this connection is the unique closed 2 -form that pulls back to the given 2-form on $\mathcal{O}_{\mu_{1}}^{-} \times \mathcal{O}_{\mu_{2}}^{-}$and for which the $L G$-action is Hamiltonian. The corresponding moment map is given by

$$
L G \times_{G}\left(\mathcal{O}_{\mu_{1}}^{-} \times \mathcal{O}_{\mu_{2}}^{-}\right) \ni\left[g, \alpha_{1}, \alpha_{2}\right] \mapsto-g \cdot\left(\alpha_{1}+\alpha_{2}\right) .
$$

For $\mu_{j}$ sufficiently small the minimal coupling form is symplectic (i.e. weakly nondegenerate).

Proposition 4.1. MW2 For $\mu_{1}, \mu_{2} \in \mathfrak{A}$ sufficiently small there is an LG-equivariant symplectomorphism

$$
\mathcal{M}\left(\Sigma_{0}^{3}\right)_{\mu_{1}, \mu_{2}, .} \cong L G \times_{G}\left(\mathcal{O}_{\mu_{1}}^{-} \times \mathcal{O}_{\mu_{2}}^{-}\right) .
$$

As a first application we recover Jeffrey's result, Theorem 1.1: If $\mu_{1}, \mu_{2}$ are small we obtain a solution of $-g \cdot\left(\alpha_{1}+\alpha_{2}\right)=\mu_{3} \in \mathfrak{A}$ with $\alpha_{j} \in \mathcal{O}_{\mu_{j}}$ only if $g$ is contained in the subgroup of constant loops, $G \subset L G$. Hence

$$
\mathcal{M}\left(\Sigma_{0}^{3}\right)_{\mu_{1}, \mu_{2}, \mu_{3}}=L G \times_{G}\left(\mathcal{O}_{\mu_{1}}^{-} \times \mathcal{O}_{\mu_{2}}^{-}\right)_{\mu_{3}}=\left(\mathcal{O}_{\mu_{1}}^{-} \times \mathcal{O}_{\mu_{2}}^{-}\right)_{\mu_{3}}=\mathcal{O}_{\mu_{1}}^{-} \times \mathcal{O}_{\mu_{2}}^{-} \times \mathcal{O}_{\mu_{3}}^{-} / / G,
$$

q.e.d.

For general $\mu_{j}$, Proposition 4.1 does not hold - in fact the induced space $L G \times{ }_{G}\left(\mathcal{O}_{\mu_{1}}^{-} \times\right.$ $\mathcal{O}_{\mu_{2}}^{-}$) will not even be symplectic if $\mu_{1}, \mu_{2} \in \mathfrak{A}$ get too big. However, just as in the finite dimensional setting (see Appendix A.3) we can make the following weaker statement: 
Let $\mathfrak{A}_{0}:=\left\{\mu \in \mathfrak{A} \mid\left\langle\alpha_{\max }, \mu\right\rangle<1\right\}$ be the set of all points in the alcove such that the isotropy group $(L G)_{\mu}$ is contained in $(L G)_{0}=G$.

Theorem 4.2. MW2 For all $\mu_{1}, \mu_{2} \in \mathfrak{A}_{0}$, there exists an LG-equivariant diffeomorphism

$$
\phi: \mathcal{M}_{I}:=\mathcal{M}\left(\Sigma_{0}^{3}\right)_{\mu_{1}, \mu_{2},} \rightarrow \mathcal{M}_{I I}:=L G \times_{G}\left(\mathcal{O}_{\mu_{1}}^{-} \times \mathcal{O}_{\mu_{2}}^{-}\right)
$$

and an LG-invariant 1-form $\beta$ on $\mathcal{M}_{I}$ such that the 2-forms $\omega_{I}, \omega_{I I}$ and the moment maps $\Phi_{I}, \Phi_{I I}$ are related by

$$
\phi^{*} \omega_{I I}=\omega_{I}+d \beta, \phi^{*} \Phi_{I I}=\Phi_{I}-\left\langle\beta,(\cdot)_{M}\right\rangle .
$$

Our method of proof in MW2 is to combine Proposition 4.1 with a DuistermaatHeckman type result, using that the equivariant cohomology classes on both sides vary linearly with $\mu_{1}, \mu_{2}$. The slopes of both changes have to be equal since for small $\mu_{j}$ the two spaces are symplectomorphic.

As we explained in Section 2, the existence of an equivariant diffeomorphism such that the equivariant 2 -forms are cohomologous implies that there is a cobordism of Hamiltonian $L G$-manifolds

$$
\mathcal{M}_{I} \sim \mathcal{M}_{I I}
$$

in the sense of Ginzburg-Guillemin-Karshon. Theorem 1.2 follows immediately by reduction: $\left(\mathcal{M}_{I}\right)_{\mu_{3}} \sim\left(\mathcal{M}_{I I}\right)_{\mu_{3}}$. We thus need to compute reductions at $\mu_{3} \in \operatorname{int}(\mathfrak{A})$ of the Hamiltonian $L G$-manifold $L G \times_{G}\left(\mathcal{O}_{\mu_{1}}^{-} \times \mathcal{O}_{\mu_{2}}^{-}\right)$. The $\mu_{3}$-level set is given by the equation

$$
-g \cdot\left(\alpha_{1}+\alpha_{2}\right)=\mu_{3} \text {. }
$$

Since $G \cdot \mathfrak{t}_{+}=\mathfrak{g}$, we may assume $-\left(\alpha_{1}+\alpha_{2}\right) \in \mathfrak{t}_{+}$. Then $g^{-1}$ maps $\mu_{3} \in \operatorname{int}(\mathfrak{A})$ to $-\left(\alpha_{1}+\alpha_{2}\right) \in \mathfrak{t}_{+}$, which implies that $g \cdot \mathfrak{A} \subset \mathfrak{t}_{+}$: In other words, $g^{-1}$ represents an element $w \in W_{\text {aff }}^{+}$with $w \mu_{3}=-\left(\alpha_{1}+\alpha_{2}\right)$. This shows that the reduction at $\mu_{3}$ is a disjoint union of all reductions $\left(\mathcal{O}_{\mu_{1}}^{-} \times \mathcal{O}_{\mu_{2}}^{-}\right)_{w \mu_{3}}$ as $w$ ranges over over $W_{\text {aff }}^{+}$. A careful check of orientations gives Theorem 1.2. We see that it is in fact sufficient that $\mu_{1}, \mu_{2} \in \mathfrak{A}_{0}$ and $\mu_{3} \in \operatorname{int}(\mathfrak{A})$.

The above argument generalizes to the case of a $b$-holed sphere with $b \geq 3$ : We have

$$
\mathcal{M}\left(\Sigma_{0}^{b}, \mu_{1}, \ldots, \mu_{b}\right) \sim \coprod_{w \in W_{\text {aff }}^{+}}(-1)^{\operatorname{length}(w)}\left(\mathcal{O}_{\mu_{1}}^{-} \times \ldots \times \mathcal{O}_{\mu_{b-1}}^{-} \times \mathcal{O}_{w \mu_{b}}^{-}\right) / / G
$$

if $\mu_{1}, \ldots, \mu_{b-1} \in \mathfrak{A}_{0}$ and $\mu_{b} \in \operatorname{int}(\mathfrak{A})$ are generic.

Remark 4.3. For the case of $G=\mathrm{SU}(n)$, the cobordism is actually a cobordism of manifolds. The reason why no orbifold singularities appear is as follows. By the holonomy description, every stabilizer $K$ for the action of $L G^{b}$ on $\mathcal{M}\left(\Sigma_{h}^{b}\right)$ is isomorphic (by the map $L G \rightarrow G, g \mapsto g(1))$ to an intersection $\bigcap_{j} Z_{g_{j}}$ of centralizers of elements in $G$. For every $k \in K$ and every $g_{j}$ in this list, $g_{j}$ is contained in $Z_{k}$, so that $Z_{g_{j}}$ contains the center of $Z_{k}$. Consequently, $K$ can only be discrete if every $Z_{k}$ is semi-simple. However, precisely for $G=\mathrm{SU}(n)$ there are no semi-simple centralizers other than $G$ itself, so that $K$ has to be equal to $Z(\mathrm{SU}(n))$. Since the cobordism is given by the product $\mathcal{M}\left(\Sigma_{0}^{3}\right)_{\mu_{1}, \mu_{2}} \times[0,1]$, 
it follows that every discrete stabilizer for the $L G$-action is $Z(G)$. Hence any reduced space at a regular value is smooth.

\section{WitTen's VOlume FORMUlas}

In this section we explain how to obtain Witten's volume formulas from the cobordism in Theorem 1.2. Since Liouville volumes of symplectic orbifolds are clearly invariants under oriented cobordism, we have the formula

$$
\operatorname{Vol}\left(\mathcal{M}\left(\Sigma_{0}^{3}, \mu_{1}, \mu_{2}, \mu_{3}\right)\right)=\sum_{w \in W_{\mathrm{aff}}^{+}}(-1)^{\operatorname{length}(w)} \operatorname{Vol}\left(\mathcal{O}_{\mu_{1}} \times \mathcal{O}_{\mu_{2}} \times \mathcal{O}_{w \mu_{3}} / / G\right)
$$

The volumes on the right hand side are given by the classical analogue (四) of Steinberg's formula discussed in Section 2. Combining (4) with (12) gives an explicit formula for the volumes of the moduli space $\mathcal{M}\left(\Sigma_{0}^{3}\right)_{\mu_{1}, \mu_{2}, \mu_{3}}$. It is convenient to replace the sum over $W_{\text {aff }}^{+}=W_{\text {aff }} / W \cong \Lambda$ by a sum over the integral lattice $\Lambda \subset W_{\text {aff }}$, using the $W$-invariance of the function $\nu \mapsto \sum_{w \in W}(-1)^{\text {length }(w)} \kappa(w \mu-\nu)$ :

Theorem 5.1. For $\mu=\left(\mu_{1}, \mu_{2}, \mu_{3}\right) \in \operatorname{int}\left(\Phi\left(\mathcal{M}\left(\Sigma_{0}^{3}\right)\right) \cap \mathfrak{A}^{3}\right)$, the Liouville volume of the moduli space $\mathcal{M}\left(\Sigma_{0}^{3}\right)_{\mu_{1}, \mu_{2}, \mu_{3}}$ is given by the formula

$$
\begin{aligned}
& \operatorname{Vol}\left(\mathcal{M}\left(\Sigma_{0}^{3}\right)_{\mu_{1}, \mu_{2}, \mu_{3}}\right) \\
& =(-1)^{\frac{1}{2} \operatorname{dim} G / T} \frac{\# Z(G)}{\operatorname{Vol}(T)} \sum_{l \in \Lambda} \sum_{w_{1}, w_{2} \in W}(-1)^{\operatorname{length}\left(w_{1} w_{2}\right)} \kappa\left(w_{1} \mu_{1}+w_{2} \mu_{2}+\mu_{3}+l\right)
\end{aligned}
$$

(where the $w_{i}$-summation is to be carried out before the summation over the integral lattice $\Lambda$ ).

Note that this holds without regularity assumptions on the $\mu_{j}$, since it is known from basic properties of DH-measures that the volume function is continuous over $\operatorname{int}\left(\Phi\left(\mathcal{M}\left(\Sigma_{0}^{3}\right)\right) \cap \mathfrak{A}^{3}\right)$, even at singular values.

This formula can be re-cast in the following form due to Witten. Let $A: T \rightarrow \mathbb{R}$ be the $W$-anti-invariant function

$$
A(\exp \xi)=\prod_{\alpha \in \Re^{+}} 2 \sin (\pi\langle\alpha, \xi\rangle) .
$$

We label the irreducible $G$-representations by their dominant weights $\lambda \in \Lambda_{+}^{*}:=\Lambda^{*} \cap \mathfrak{t}_{+}$ and let $\chi_{\lambda}: G \rightarrow \mathbb{C}$ denote the character and $d_{\lambda}=\chi_{\lambda}(e)$ the dimension.

Theorem 5.2. (Witten formula for the three-holed sphere.) For $\mu=\left(\mu_{1}, \mu_{2}, \mu_{3}\right) \in$ $\operatorname{int}\left(\Phi\left(\mathcal{M}\left(\Sigma_{0}^{3}\right)\right) \cap \mathfrak{A}^{3}\right)$, the volume of the moduli space of the three-holed sphere $\mathcal{M}\left(\Sigma_{0}^{3}\right)_{\mu_{1}, \mu_{2}, \mu_{3}}$ is given by

$$
\operatorname{Vol}\left(\mathcal{M}\left(\Sigma_{0}^{3}\right)_{\mu_{1}, \mu_{2}, \mu_{3}}\right)=\# Z(G) \frac{\operatorname{Vol}(G)}{\operatorname{Vol}(T)^{3}} \prod_{j=1}^{3} A\left(e^{\mu_{j}}\right) \sum_{\lambda \in \Lambda_{+}^{*}} \frac{1}{d_{\lambda}} \prod_{j=1}^{3} \chi_{\lambda}\left(e^{\mu_{j}}\right) .
$$


Here $\operatorname{Vol}(T)$ and $\operatorname{Vol}(G)$ are the Riemannian volumes with respect to the normalized inner product on $\mathfrak{g}$.

Proof. Note first that the right hand sides of both (14) and (13) define naturally $W_{\text {aff- }}$ anti-invariant tempered distributions in $\mu_{1}, \mu_{2}, \mu_{3} \in \mathfrak{t}$. It is sufficient to show that we get the same answer if we apply the constant coefficient differential operator

$$
\mathcal{D}_{3}=\prod_{\alpha \in \mathfrak{R}^{+}}\left\langle\alpha, \frac{\partial}{\partial \mu_{3}}\right\rangle .
$$

to both expressions. (We use the fact that $\mathcal{D}_{3} u=0$ has no $W$-anti-invariant solutions in the space of tempered distributions.) The measure $\kappa$ satisfies $\mathcal{D}_{3} \kappa\left(\mu_{3}\right)=\delta\left(\mu_{3}\right)$. Using the Poisson summation formula, we obtain:

$$
\begin{aligned}
\frac{1}{\# Z(G)} \mathcal{D}_{3} \operatorname{Vol}\left(\mathcal{M}\left(\Sigma_{0}^{3}\right)_{\mu_{1}, \mu_{2}, \mu_{3}}\right) \\
=\frac{(-1)^{\frac{1}{2} \operatorname{dim} G / T}}{\operatorname{Vol}(T)} \sum_{l \in \Lambda} \sum_{w_{1}, w_{2} \in W}(-1)^{\operatorname{length}\left(w_{1} w_{2}\right)} \delta\left(w_{1} \mu_{1}+w_{2} \mu_{2}+\mu_{3}+l\right) \\
=\frac{(-1)^{\frac{1}{2} \operatorname{dim} G / T}}{\operatorname{Vol}(T)^{2}} \sum_{\lambda \in \Lambda^{*}} \sum_{w_{1}, w_{2} \in W}(-1)^{\operatorname{length}\left(w_{1} w_{2}\right)} e^{2 \pi i\left\langle w_{1} \mu_{1}+w_{2} \mu_{2}+\mu_{3}, \lambda\right\rangle} .
\end{aligned}
$$

In this sum, weights $\lambda \in \Lambda^{*}$ which lie on a wall of some Weyl chamber do not contribute because

$$
\sum_{w \in W}(-1)^{\operatorname{length}(w)} e^{2 \pi i\langle w \nu, \lambda\rangle}=0
$$

if $\lambda$ has a non-trivial stabilizer in $W$. Since $\Lambda^{*} \cap \operatorname{int}\left(\mathfrak{t}_{+}\right)=\rho+\Lambda_{+}^{*}$, we can rewrite the sum as

$$
\begin{aligned}
& \frac{(-1)^{\frac{1}{2} \operatorname{dim} G / T}}{\operatorname{Vol}(T)^{2}} \sum_{\lambda \in \Lambda_{+}^{*}} \sum_{w_{1}, w_{2}, w \in W}(-1)^{\operatorname{length}\left(w_{1} w_{2}\right)} e^{2 \pi i\left\langle w_{1} \mu_{1}+w_{2} \mu_{2}+\mu_{3}, w(\lambda+\rho)\right\rangle} \\
= & \frac{(-1)^{\frac{1}{2} \operatorname{dim} G / T}}{\operatorname{Vol}(T)^{2}} \sum_{\lambda \in \Lambda_{+}^{*}} \sum_{w_{1}, w_{2}, w_{3} \in W}(-1)^{\operatorname{length}\left(w_{1} w_{2}\right)} e^{2 \pi i\left\langle w_{1} \mu_{1}+w_{2} \mu_{2}+w_{3} \mu_{3}, \lambda+\rho\right\rangle} \\
= & \mathcal{D}_{3} \frac{(-1)^{\frac{1}{2} \operatorname{dim} G / T}}{\operatorname{Vol}(T)^{2}} \sum_{\lambda \in \Lambda_{+}^{*}} \frac{(2 \pi i)^{-\frac{1}{2} \operatorname{dim} G / T}}{\prod_{\alpha \in \mathfrak{R}^{+}}\langle\alpha, \lambda+\rho\rangle} \prod_{j=1}^{3}\left(\sum_{w_{j} \in W}(-1)^{\operatorname{length}\left(w_{j}\right)} e^{2 \pi i\left\langle w_{j} \mu_{j}, \lambda+\rho\right\rangle}\right) .
\end{aligned}
$$

The last expression is identified with the right hand side of (14), using the Weyl character formula

$$
\chi_{\lambda}(\exp \xi)=\frac{\sum_{w \in W}(-1)^{\text {length }(w)} e^{2 \pi i\langle w(\lambda+\rho), \xi\rangle}}{i^{\frac{1}{2} \operatorname{dim} G / T} A\left(e^{\xi}\right)}
$$


and the Weyl dimension formula

$$
d_{\lambda}=\frac{\prod_{\alpha \in \mathfrak{R}^{+}}\langle\alpha, \lambda+\rho\rangle}{\prod_{\alpha \in \mathfrak{R}^{+}}\langle\alpha, \rho\rangle}=(2 \pi)^{\frac{1}{2} \operatorname{dim} G / T} \operatorname{Vol}(G / T) \prod_{\alpha \in \mathfrak{R}^{+}}\langle\alpha, \lambda+\rho\rangle .
$$

Here $\operatorname{Vol}(G / T)$ is the Riemannian volume of $G / T$ with respect to the inner product on $\mathfrak{g}$ (see e.g. $\mathrm{BGV})$.

Remark 5.3. The differential equation for $\mathcal{D}_{3} \operatorname{Vol}\left(\mathcal{M}\left(\Sigma_{0}^{3}\right)_{\mu_{1}, \mu_{2}, \mu_{3}}\right)$ appearing in this proof is interpreted in Jeffrey-Weitsman [JW2 in terms of symplectic volumes of intersections of divisors in the moduli space.

From the volume formula for the three-holed sphere, formulas for the general case are obtained by gluing. For this we need:

Proposition 5.4. Let $\Sigma=\Sigma_{h}^{b}$ be obtained from a possibly disconnected 2-manifold $\hat{\Sigma}$ by gluing two boundary components $B_{ \pm} \subset \partial \Sigma$. Suppose $\mu=\left(\mu_{1}, \ldots, \mu_{b}\right) \in$ int $\mathfrak{A}^{b}$ is such that $\mathcal{M}\left(\Sigma, \mu_{1}, \ldots, \mu_{b}\right)$ contains at least one connection with stabilizer $Z(G)$. Then

$$
\operatorname{Vol}\left(\mathcal{M}\left(\Sigma, \mu_{1}, \ldots, \mu_{b}\right)\right)=\frac{1}{k} \int_{\mathfrak{A}} \operatorname{Vol}\left(\mathcal{M}\left(\hat{\Sigma}, \mu_{1}, \ldots, \mu_{b}, \nu, * \nu\right)\right)|d \nu|
$$

Here the measure $|d \nu|$ on $\mathfrak{A} \subset \mathfrak{t}$ is the normalized measure for which $\mathfrak{t} / \Lambda^{*}$ has measure 1 , and $k=1$ if $\hat{\Sigma}$ is connected and equal to $\# Z(G)$ if $\hat{\Sigma}$ is disconnected.

This is proved in Jeffrey-Weitsman JW2]. It also follows from the "Gluing equals Reduction" principle, Theorem 3.2 since the proof of Theorem A.3 goes through for Hamiltonian loop group actions with proper moment maps ( $\mathbb{M W} \mathbb{1}$, Proposition 3.12).

The reason for the factor $\frac{1}{k}$ is that the generic stabilizer for the $L G \times L G$-action on $\mathcal{M}\left(\hat{\Sigma}, \mu_{1}, \ldots, \mu_{b}, \cdot, \cdot\right)$ is $Z(G)$ if $\hat{\Sigma}$ is connected, $Z(G) \times Z(G)$ otherwise. Carrying out the integrations gives:

Proposition 5.5. Suppose $2 h+b \geq 3$. For all $\mu_{1}, \ldots \mu_{b} \in \operatorname{int}(\mathfrak{A})$ such that $\mathcal{M}\left(\Sigma_{h}^{b}, \mu_{1}, \ldots, \mu_{b}\right)$ contains the gauge equivalence class of at least one connection with stablizer $Z(G)$, the Liouville volume of the moduli space $\mathcal{M}\left(\Sigma_{h}^{b}, \mu_{1}, \ldots, \mu_{b}\right)$ is given by the formula

$$
\operatorname{Vol}\left(\mathcal{M}\left(\Sigma_{h}^{b}, \mu_{1}, \ldots, \mu_{b}\right)\right)=\# Z(G) \frac{\operatorname{Vol}(G)^{2 h-2+b}}{\operatorname{Vol}(T)^{b}} \prod_{j=1}^{b} A\left(e^{\mu_{j}}\right) \sum_{\lambda \in \Lambda_{+}^{*}} \frac{1}{d_{\lambda}^{2 h-2+b}} \prod_{j=1}^{b} \chi_{\lambda}\left(e^{\mu_{j}}\right) .
$$

Proof. This follows from Proposition 5.4 and (14) by iterated gluing of 3-holed spheres. We have to use that $A\left(e^{\xi}\right) A\left(e^{* \xi}\right)=\left|A\left(e^{\xi}\right)\right|^{2}$ and that by Weyl's integration formula and the orthogonality relations of irreducible characters,

$$
\int_{\mathfrak{A}} \chi_{\lambda_{1}}\left(e^{\xi}\right) \chi_{\lambda_{2}}\left(e^{\xi}\right)\left|A\left(e^{\xi}\right)\right|^{2}|\mathrm{~d} \xi|=\left\{\begin{array}{cc}
1 & \text { if } \lambda_{1}=* \lambda_{2} \\
0 & \text { otherwise }
\end{array}\right.
$$


where $|\mathrm{d} \xi|$ is the measure on $\mathfrak{t}$ which is normalized with respect to the lattice $\Lambda$. In Proposition 5.4 we need to use instead Lebesgue measure normalized with respect to $\Lambda^{*}$. Since these two measures differ by a factor $\operatorname{Vol}(T)^{2}$ (where $\operatorname{Vol}(T)$ is the volume of $T$ with respect to the inner product on $\mathfrak{t} \subset \mathfrak{g}$ ), we get an overall factor $\operatorname{Vol}(T)^{2(3 h-3+b)}$ from the $3 h-3+b$ gluing circles, which combines with the factor $\operatorname{Vol}(T)^{-3(2 h-2+b)}$ corresponding to the number of 3 -holed spheres to a factor $\operatorname{Vol}(T)^{-b}$.

Remark 5.6. The moduli space $\mathcal{M}\left(\Sigma_{h}^{0}\right)$ for a surface without boundary and genus $h \geq 2$ always contains a connection with stabilizer equal to $Z(G)$.

Finally, we would like to get rid of the assumption that the $\mu_{j}$ lie in the interior of the fundamental alcove. For $\nu=\left(\nu_{1}, \ldots, \nu_{b}\right) \in \mathfrak{A}^{b}$ such that $\Phi^{-1}(\nu)$ contains a connection with stabilizer $Z(G)$, we have by Appendix A.1, Equation (19)

$$
\operatorname{Vol} \mathcal{M}\left(\Sigma_{h}^{b}, \nu_{1}, \ldots, \nu_{b}\right)=\lim _{\substack{\mu_{j} \rightarrow \nu_{j} \\ \mu_{j} \in \operatorname{int}(\mathfrak{A})}} \frac{\operatorname{Vol} \mathcal{M}\left(\Sigma_{h}^{b}, \mu_{1}, \ldots, \mu_{b}\right)}{\operatorname{Vol}\left(K_{1} \cdot\left(\mu_{1}-\nu_{1}\right)\right) \ldots \operatorname{Vol}\left(K_{b} \cdot\left(\mu_{b}-\nu_{b}\right)\right)}
$$

where $K_{j}=(L G)_{\nu_{j}}$. Note that $K_{j}$ contains the maximal torus $T$ of $G$. A natural choice of positive Weyl chamber for $K_{j}$ is given by $\mathfrak{t}_{+, j}^{*}=\mathbb{R}_{+} \cdot\left(\mathfrak{A}-\nu_{j}\right)$, and the corresponding set $\mathfrak{R}_{j}^{+}$of positive roots of $K_{j}$ is the set of all roots $\alpha$ of $G$ such that $\left\langle\alpha, \nu_{j}\right\rangle \in\{0,-1\}$.

The Liouville volume of a coadjoint orbit $K_{j} \cdot\left(\mu_{j}-\nu_{j}\right)$ is related to the Riemannian volume of $K_{j} / T$ by

$$
\operatorname{Vol}\left(K_{j} \cdot\left(\mu_{j}-\nu_{j}\right)\right)=(2 \pi)^{\frac{1}{2} \operatorname{dim} K_{j} / T} \prod_{\alpha \in \mathfrak{R}_{j}^{+}}\left\langle\alpha, \mu_{j}-\nu_{j}\right\rangle \operatorname{Vol}\left(K_{j} / T\right)
$$

(see e.g. [BGV]). Since $K_{j} \cong Z_{\exp \left(\mu_{j}\right)}$ the factor $\operatorname{Vol}\left(K_{j} / T\right)$ combines with $\operatorname{Vol}(G / T)^{-1}$ to give the Riemannian volume of the conjugacy class $\mathcal{C}_{\mu_{j}}=G / Z_{\exp \left(\mu_{j}\right)}$. Moreover

$$
\lim _{\mu_{j} \rightarrow \nu_{j}} \frac{\prod_{\alpha \in \mathfrak{R}^{+}} 2 \sin \left(\pi\left\langle\alpha, \mu_{j}\right\rangle\right)}{\prod_{\alpha \in \mathfrak{R}_{j}^{+}} 2 \pi\left\langle\alpha, \mu_{j}-\nu_{j}\right\rangle}=\prod_{\substack{\alpha \in \mathfrak{R}^{+} \\\left\langle\alpha, \nu_{j}\right\rangle \notin\{0,1\}}} 2 \sin \left(\pi\left\langle\alpha, \nu_{j}\right\rangle\right)
$$

so that we obtain:

Theorem 5.7 (Witten Formula). Suppose $2 h+b \geq 3$. Let $\mu=\left(\mu_{1}, \ldots, \mu_{b}\right) \in \mathfrak{A}^{b}$ be such that the level set $\Phi^{-1}(\mu)$ contains a connection with stabilizer $Z(G)$. The volume of the moduli space of the 2-manifold $\Sigma_{h}^{b}$ with fixed holonomies $\mu_{1}, \ldots, \mu_{b}$ is given by the formula

$$
\begin{aligned}
& \operatorname{Vol}\left(\mathcal{M}\left(\Sigma_{h}^{b}, \mu_{1}, \ldots, \mu_{b}\right)\right) \\
& =\# Z(G) \operatorname{Vol}(G)^{2 h-2} \prod_{j=1}^{b}\left(\operatorname{Vol}\left(\mathcal{C}_{\mu_{j}}\right) \prod_{\substack{\alpha \in \Re+\\
\left\langle\alpha, \mu_{j}\right\rangle \notin\{0,1\}}} 2 \sin \left(\pi\left\langle\alpha, \mu_{j}\right\rangle\right)\right) \sum_{\lambda \in \Lambda_{+}^{*}} \frac{1}{d_{\lambda}^{2 h-2+b}} \prod_{j=1}^{b} \chi_{\lambda}\left(e^{\mu_{j}}\right)
\end{aligned}
$$


In particular,

$$
\operatorname{Vol}\left(\mathcal{M}\left(\Sigma_{h}^{0}\right)\right)=\# Z(G) \operatorname{Vol}(G)^{2 h-2} \sum_{\lambda \in \Lambda_{+}^{*}} \frac{1}{d_{\lambda}^{2 h-2}}
$$

The above formulas can be made more explicit for $G=S U(2)$. Viewed as an element of $\mathfrak{t}$, the exponential of $\rho$ is the diagonal matrix $-I \in Z(\mathrm{SU}(2))$ and the fundamental alcove is the interval $[0, \rho]$. Since $\rho=\frac{\alpha}{2}$ has length $\frac{1}{\sqrt{2}}$, we have $\operatorname{Vol}(T)=\sqrt{2}$. Also, $\operatorname{Vol}(\mathrm{SU}(2) / T)=\frac{1}{2 \pi}$ and $\# Z(G)=2$. The character of the $k$-dimensional representation is given by

$$
\chi_{k}(\exp (t \rho))=\frac{\sin (\pi k t)}{\sin (\pi t)} .
$$

For $b=0$ the above formula reads

$$
\operatorname{Vol}\left(\mathcal{M}\left(\Sigma_{h}^{0}\right)\right)=\frac{2^{h}}{(2 \pi)^{2 h-2}} \zeta(2 h-2) .
$$

In particular, $\operatorname{Vol}\left(\mathcal{M}\left(\Sigma_{2}^{0}\right)\right)=\frac{1}{\pi^{2}} \zeta(2)=\frac{1}{6}$ which matches with the well-known fact $\mathcal{M}\left(\Sigma_{2}^{0}\right) \cong \mathbb{C} P(3)$. For $b=1, \mu=t \rho$ we have to distinguish the three cases $0<t<1$ and $t=0,1$. For $t=0$ we have $\operatorname{Vol}\left(\mathcal{M}\left(\Sigma_{h}^{1}, 0\right)\right)=\operatorname{Vol}\left(\mathcal{M}\left(\Sigma_{h}^{0}\right)\right)$ as expected. For $t=1$ we find $\chi_{k}(\exp \rho)=(-1)^{k+1} k$ and therefore

$$
\operatorname{Vol}\left(\mathcal{M}\left(\Sigma_{h}^{1}, \rho\right)\right)=\frac{2^{h}}{(2 \pi)^{2 h-2}} \sum_{k=1}^{\infty} \frac{(-1)^{k+1}}{k^{2 h-2}}=\frac{2^{h}-2^{2-h}}{(2 \pi)^{2 h-2}} \zeta(2 h-2) .
$$

For $0<t<1$ we have

$$
\operatorname{Vol}\left(\mathcal{M}\left(\Sigma_{h}^{1}, t \rho\right)\right)=2^{h} \sum_{k=1}^{\infty} \frac{1}{(2 \pi k)^{2 h-1}} \sin (\pi k t) .
$$

The Fourier summation gives Bernoulli polynomials, see [D2. In particular, for $h=1$ the result is the sawtooth function

$$
\operatorname{Vol}\left(\mathcal{M}\left(\Sigma_{1}^{1}, t \rho\right)\right)=2\left(\frac{t}{2}-\left[\frac{t}{2}\right]\right)
$$

\section{Appendix A. Background on Symplectic Reduction}

In this appendix we summarize some basic facts about reduction of Hamiltonian $K$ manifolds, where $K$ is a compact connected Lie group. Let $T \subset K$ be the maximal torus of $K$, and $\mathfrak{t}_{+}^{*} \subset \mathfrak{t}^{*} \subset \mathfrak{k}^{*}$ a closed fundamental Weyl chamber. Thus $\mathfrak{t}_{+}^{*}=\mathfrak{k}^{*} / K$ parametrizes the set of coadjoint orbits in $\mathfrak{k}^{*}$. The stabilizer $K_{\mu}$ of a point $\mu \in \mathfrak{t}_{+}^{*}$ depends only on the open face of $\mathfrak{t}_{+}^{*}$ containing $\mu$; in particular $K_{\mu}=T$ for $\mu \in \operatorname{int}\left(\mathfrak{t}_{+}^{*}\right)$. 
A.1. Singular reduced spaces. We start with a brief discussion of singular symplectic quotients. Most of this material is due to Sjamaar-Lerman [SD]. Suppose that $(M, \omega, \Phi)$ is a connected symplectic Hamiltonian $K$-manifold ( $\operatorname{dim} M=2 n$ ) with proper moment map. Decomposing $M$ according to conjugacy classes of stabilizer groups, and then decomposing further into connected components gives the orbit type stratification

$$
M=\cup M_{\lambda}
$$

There exists a unique principal stratum $M^{\text {prin }}$ which is open and dense; the corresponding stabilizer group $\Gamma \subset K$ (defined up to conjugacy) is called the principal stabilizer. Suppose now that $\mu \in \mathfrak{k}^{*}$ is a possibly singular value of $\Phi$. Using local normal forms one can show that every intersection $\Phi^{-1}(\mu) \cap M_{\lambda}$ is smooth, and the decomposition

$$
\Phi^{-1}(\mu)=\cup_{\lambda} \Phi^{-1}(\mu) \cap M_{\lambda}
$$

makes $\Phi^{-1}(\mu)$ into a stratified singular space. Moreover, taking the quotient by $K_{\mu}$ one obtains a stratification of the reduced space $M_{\mu}=\Phi^{-1}(\mu) / K_{\mu}$ all of whose strata are symplectic manifolds. It is shown in SL that every $M_{\mu}$ has a unique open, dense connected principal stratum $M_{\mu}^{\text {prin }}$. If $\Phi^{-1}(\mu)$ meets $M^{\text {prin }}$ then

$$
M_{\mu}^{\text {prin }}=\left(\Phi^{-1}(\mu) \cap M^{\text {prin }}\right) / K_{\mu} .
$$

The local structure of the singular space $M_{\mu}$ is that of an iterated symplectic cone (see [SD], section 6), which implies in particular that the singular strata have finite Liouville volume. One defines $\operatorname{Vol}\left(M_{\mu}\right)$ to be the Liouville volume of $M_{\mu}^{\text {prin }}$.

Let us now assume that the generic stabilizer $\Gamma$ is discrete, which implies that $\Phi \mid M^{\text {prin }}$ is a submersion. From the Liouville form $\omega^{n} / n$ ! and any translation invariant volume form on $\mathfrak{k}^{*}$, the level sets $\Phi^{-1}(\mu) \cap M^{\text {prin }}$ acquire natural volume forms and orientations. Their volume $\operatorname{Vol}\left(\Phi^{-1}(\mu)\right):=\operatorname{Vol}\left(\Phi^{-1}(\mu) \cap M^{\text {prin }}\right)$ is related to the volume of the reduced space $\operatorname{Vol}\left(M_{\mu}\right)$ by

$$
\operatorname{Vol}\left(\Phi^{-1}(\mu)\right)=\frac{\operatorname{Vol}\left(M_{\mu}\right)}{\operatorname{Vol} K \cdot \mu} \frac{\operatorname{Vol}(K)}{\# \Gamma}
$$

where $\operatorname{Vol}(K \cdot \mu)$ is the symplectic volume of the coadjoint orbit through $\mu$, and $\operatorname{Vol}(K)$ the volume of $K$ with respect to the dual measure on $\mathfrak{k}$. The function $\mu \mapsto \operatorname{Vol}\left(\Phi^{-1}(\mu)\right)$ is continuous over $\Phi\left(M^{\text {prin }}\right)$. Combining this with $\operatorname{Vol}(K \cdot \mu) / \operatorname{Vol}(K \cdot \nu)=\operatorname{Vol}\left(K_{\nu} \cdot(\mu-\nu)\right)$, the symplectic volume of the coadjoint $K_{\nu}$-orbit through $\mu-\nu \in \mathfrak{k}_{\nu}^{*}$, it follows that for any $\nu \in \Phi\left(M^{\text {prin }}\right) \cap \mathfrak{t}_{+}^{*}$,

$$
\operatorname{Vol}\left(M_{\nu}\right)=\lim _{\mu \rightarrow \nu} \frac{\operatorname{Vol}\left(M_{\mu}\right)}{\operatorname{Vol}\left(K_{\nu} \cdot(\mu-\nu)\right)} .
$$

This is particularly useful since it allows to compute volumes of singular reduced spaces as limits of volumes of regular reduced spaces. We emphasize that (19) does not hold if $\nu \in \mathfrak{t}_{+}^{*}$ is not contained in $\Phi\left(M^{\text {prin }}\right)$. 
A.2. Duistermaat-Heckman. Suppose that $H$ is another compact Lie group and that $(M, \omega,(\Phi, \Psi))$ is a compact Hamiltonian $K \times H$-manifold. Let $\sigma$ be an open face of $\mathfrak{t}_{+}^{*}$. The tangent space to $\sigma$ is given by $\mathfrak{z}\left(K_{\sigma}\right)^{*}$, the dual of the Lie algebra of the center of $K_{\sigma}$. Let $\Phi(M)_{\text {reg }}$ be the set of regular values of $\Phi$.

Theorem A.1 (Duistermaat-Heckman). Let $M$ be a compact Hamiltonian $K \times H$ manifold with moment map $(\Phi, \Psi)$. The $K$-reduced spaces $M_{\mu}$ for $\mu$ in a connected component of the set $\sigma \cap \Phi(M)_{\text {reg }}$ are $H$-equivariantly diffeomorphic, and the cohomology class of the H-equivariant 2-forms $\tilde{\omega}_{\mu}$ varies linearly: That is,

$$
\left[\tilde{\omega}_{\mu_{1}}\right]-\left[\tilde{\omega}_{\mu_{2}}\right]=\left\langle\mu_{1}-\mu_{2}, \tilde{c}\right\rangle
$$

for a fixed class $\tilde{c} \in H_{H}^{*}\left(M_{\mu_{1}}\right) \otimes \mathfrak{z}\left(K_{\sigma}\right)$.

An immediate consequence of the DH-theorem is that if $(M, \omega, \Phi)$ is a compact symplectic Hamiltonian $K$-manifold, the function $\mu \mapsto \operatorname{Vol}\left(M_{\mu}\right)$ is given by a polynomial on every connected component of $\sigma \cap \Phi(M)_{\text {reg }}$.

Note that in Theorem A.1 we did not require that $\omega$ be symplectic. The result is a consequence of two facts in equivariant cohomology: First, for any compact Hamiltonian $K \times H$-manifold $(M, \omega,(\Phi, \Psi))$, with 0 a regular value of $\Phi$, the equivariant cohomology class of $\tilde{\omega}_{0}$ is the image of the $K \times H$-equivariant class of $\tilde{\omega}$ under the map

$$
H_{K \times H}^{*}(M) \rightarrow H_{K \times H}^{*}\left(\Phi^{-1}(0)\right) \rightarrow H_{H}^{*}(M / / K) .
$$

Second, for any regular value $\mu$ of $\Phi$, zero is a regular value for the diagonal action on $M \times \mathcal{O}_{\mu}^{-}$, and $M_{\mu}=M \times O_{\mu}^{-} / / K$. Clearly, as $\mu$ varies in $\sigma$ the equivariant cohomology class of the equivariant 2 -form on $M \times \mathcal{O}_{\mu}^{-} \cong M \times K / K_{\sigma}$ varies linearly.

A.3. Symplectic Fibrations. Suppose that $(M, \omega,(\Phi, \Psi))$ is a compact a Hamiltonian $K \times H$-manifold. Let $\sigma, \tau$ be open faces of $\mathfrak{t}_{+}^{*}$ with $\sigma \subset \bar{\tau}$, and let $\nu \in \sigma$ and $\mu \in \tau$ regular values of $\Phi$, contained in the same connected component of $\Phi(M)_{\text {reg }}$. Choosing an $H$-equivariant diffeomorphism $\Phi^{-1}(\mu) \cong \Phi^{-1}(\nu)$ makes $M_{\mu}$ into a fiber bundle over $M_{\nu}$, with fiber the coadjoint orbit $K_{\nu} / K_{\mu} \cong K_{\nu} \cdot(\mu-\nu) \subset \mathfrak{k}_{\nu}^{*}$ :

$$
M_{\mu} \cong \Phi^{-1}(\nu) \times_{K_{\nu}}\left(K_{\nu} \cdot(\mu-\nu)\right)^{-} .
$$

Let $\theta \in \Omega^{1}\left(\Phi^{-1}(\nu), \mathfrak{k}_{\nu}\right)^{K_{\nu}}$ be an $H$-invariant principal connection, and let $\sigma_{\nu, \mu}$ be the KKS-form on $K_{\nu} \cdot(\mu-\nu)$. The closed 2-form on $\Phi^{-1}(\nu) \times K_{\nu} \cdot(\mu-\nu)$ given by

$$
\iota_{\nu}^{*} \omega-\sigma_{\nu, \mu}-\mathrm{d}\langle\alpha, \theta\rangle
$$

(where $\iota_{\nu}: \Phi^{-1}(\nu) \rightarrow M$ and $\alpha: K_{\nu} \cdot(\mu-\nu) \rightarrow \mathfrak{k}_{\nu}^{*}$ are the embeddings) is basic, and therefore descends to a closed 2 -form on the associated bundle $\Phi^{-1}(\nu) \times_{K_{\nu}}\left(K_{\nu} \cdot(\mu-\nu)\right)^{-}$ known as the minimal coupling form of Sternberg [S]. It is easy to see that the $H$-action on this bundle is Hamiltonian, with moment map naturally induced from $\Psi$.

Theorem A.2 (Fibrations of Reduced Spaces). Let the associated bundle

$$
\Phi^{-1}(\nu) \times_{K_{\nu}}\left(K_{\nu} \cdot(\mu-\nu)\right)^{-} \rightarrow M_{\nu}
$$


be equipped with the minimal coupling form for an $H$-invariant principal connection $\theta \in$ $\Omega^{1}\left(\Phi^{-1}(\nu), \mathfrak{k}_{\nu}\right)$. The H-equivariant closed 2-form on $M_{\mu}$ is equivariantly cohomologous to the equivariant minimal coupling form. If $\omega$ is symplectic and $\mu$ sufficiently close to $\nu$, the minimal coupling form is symplectic, and $M_{\mu}$ is equivariantly symplectomorphic the associated bundle $\Phi^{-1}(\nu) \times_{K_{\nu}}\left(K_{\nu} \cdot(\mu-\nu)\right)^{-}$.

Symplectic fibrations by coadjoint orbits are studied in great detail in the book [GLS.

A.4. Diagonal reduction. Suppose now that $\left(M, \omega,\left(\Phi_{+}, \Phi_{-}\right)\right)$is a compact connected symplectic Hamiltonian $K \times K$-manifold. (Typically $M$ is the direct product of two Hamiltonian $K$-manifolds $\left(M_{ \pm}, \omega_{ \pm}, \Phi_{ \pm}\right)$.) Suppose also that the generic stabilizers $\Gamma_{\text {diag }} \subset \operatorname{diag}(K)$ for the diagonal $K$-action on $\Phi_{\text {diag }}^{-1}(0)$ and $\Gamma \subset K \times K$ for the $K \times K$ action on $M$ are discrete. Let $*: \mathfrak{t}_{+}^{*} \rightarrow \mathfrak{t}_{+}^{*}$ be the involution defined by

$$
K \cdot(* \mu):=K \cdot(-\mu) \cong(K \cdot \mu)^{-} \text {. }
$$

Theorem A.3. The symplectic volume of $M / / \operatorname{diag}(K)$ is related to the volumes of the $K \times K$-reduced spaces by

$$
\operatorname{Vol}(M / / \operatorname{diag}(K))=\operatorname{Vol}(T) \frac{\# \Gamma}{\# \Gamma_{\text {diag }}} \int_{t_{+}^{*}} \operatorname{Vol}\left(M_{\mu, * \mu}\right)|d \mu| .
$$

Here $|d \mu|$ is any choice of Lebesgue measure on $\mathfrak{t}^{*}$ and $\operatorname{Vol}(T)$ the volume of the torus with respect to the induced measure on $\mathfrak{t}$.

Notice that this result is more or less obvious if $K=T$ is a torus. The general case reduces to the abelian case as follows: By the Guillemin-Sternberg symplectic crosssection theorem, the subset $Y=\left(\Phi_{+}, \Phi_{-}\right)^{-1}\left(\operatorname{int}\left(\mathfrak{t}_{+}^{*}\right) \times-\operatorname{int}\left(\mathfrak{t}_{+}^{*}\right)\right)$ is a smooth symplectic submanifold of $M$, and is a Hamiltonian $T \times T$-manifold with the restriction of $\left(\Phi_{+}, \Phi_{-}\right)$ as a moment map. Moreover, $(K \times K) \cdot Y$ is open and dense in $M$ which implies that $Y / / \operatorname{diag}(T) \subset M / / \operatorname{diag}(K)$ is open and dense.

\section{REFERENCES}

[A] M. F. Atiyah: The geometry and physics of knots. Cambridge University Press, Cambridge, 1990

[AB] M. F. Atiyah, R. Bott: The Yang-Mills equations over Riemann surfaces. Phil. Trans. R. Soc. London 308 (1982), 523-615.

[BGV] N. Berline, E. Getzler, M. Vergne: Heat kernels and Dirac operators. Grundlehren der Mathematischen Wissenschaften 298. Springer-Verlag, Berlin, 1992.

[BrD] T. Bröcker, T. tom Dieck: Representations of compact Lie groups. Graduate Texts in Mathematics, 98. Springer-Verlag, New York, 1985.

[C] S. Chang: In preparation.

[D1] S. K. Donaldson: Boundary value problems for Yang-Mills fields. J. Geom. Phys. 8 (1992), 89-122.

[D2] S. K. Donaldson: Gluing techniques in the cohomology of moduli spaces. Topological methods in modern mathematics (Stony Brook, NY, 1991), 137-170, Publish or Perish, Houston, TX, 1993

[GGK] V. Ginzburg, V. Guillemin, Y. Karshon: Cobordism theory and localization formulas for Hamiltonian group actions. Int. Math. Res. Notices 5 (1996), 221-234.

[GS] V. Guillemin, S. Sternberg: Symplectic techniques in physics. Cambridge University Press, 1990. 
[GLS] V. Guillemin, E. Lerman, S. Sternberg: Symplectic fibrations and multiplicity diagrams. Cambridge University Press, Cambridge, 1996.

[GP] V. Guillemin, E. Prato: Heckman, Kostant, and Steinberg formulas for symplectic manifolds. Adv. Math. 82 (1990), 160-179.

[J] L. Jeffrey: Extended moduli spaces of flat connections on Riemann surfaces. Math. Ann. 298 (1994), 667-692.

[JK] L. Jeffrey, F. Kirwan: Intersection pairings in moduli spaces of vector bundles of arbitrary rank over a Riemann surface. Preprint (1996), alg-geom/9608029.

[JW1] L. Jeffrey, J. Weitsman: Toric structures on the moduli space of flat connections on a Riemann surface. II. Inductive decomposition of the moduli space. Math. Ann. 307 (1997), 93-108.

[JW2] L. Jeffrey, J. Weitsman: Symplectic geometry of the moduli space of flat connections on a Riemann surface: inductive decompositions and vanishing theorems. Preprint, December 1996.

[L1] K. Liu: Heat kernel and moduli space. Math. Res. Lett. 3 (1996), 743-76.

[L2] K. Liu: Heat kernel and moduli spaces II Preprint, dg-ga/9612001

[M] S. Martin: Private communication.

[MW1] E. Meinrenken, C. Woodward: A symplectic proof of Verlinde factorization. Preprint, August 1996. dg-ga/9612018

[MW2] E. Meinrenken, C. Woodward: Fusion of Hamiltonian loop group manifolds and cobordism. Preprint, July 1997.

[PS] A. Pressley, G. Segal: Loop groups. Oxford University Press, Oxford, 1988.

[Se] G. Segal: Lecture notes, Oxford, 1988.

[S] S. Sternberg: Minimal coupling and the symplectic mechanics of a classical particle in the presence of a Yang-Mills field. Proc. Nat. Acad. Sci. U.S.A. 74 (1977), 5253-5254.

[SL] R. Sjamaar, R. Lerman: Stratified symplectic spaces and reduction. Ann. of Math. 134 (1991), $375-422$.

[T] M. Thaddeus: Stable pairs, linear systems and the Verlinde formula. Invent. Math. 117 (1994), $317-353$.

[V] E. Verlinde: Fusion rules and modular transformations in $2 d$ conformal field theory. Nuclear Physics B 300 (1988), 360-376.

[W1] E. Witten: On quantum gauge theories in two dimensions. Comm. Math. Phys. 141 (1991), 153-209.

[W2] E. Witten: Two-dimensional gauge theories revisited. J. Geom. Phys. 9 (1992), 303-368.

[W3] E. Witten: The Verlinde algebra and the cohomology of the Grassmannian. Geometry, topology, and physics, 357-422, Conf. Proc. Lecture Notes Geom. Topology, VI, Internat. Press, Cambridge, 1995.

Massachusetts Institute of Technology, Department of Mathematics, Cambridge, MASSACHUSETTS 02139

E-mail address: mein@math.mit.edu

Harvard University, Department of Mathematics, 1 Oxford Street, Cambridge, MasSACHUSETTS 02138

E-mail address: woodward@math.harvard.edu 